PROCEEDINGS OF THE

AMERICAN MATHEMATICAL SOCIETY

Volume 126, Number 2, February 1998, Pages 531-534

S 0002-9939(98)04201-4

\title{
EVERY COMPLETE DOUBLING METRIC SPACE CARRIES A DOUBLING MEASURE
}

\author{
JOUNI LUUKKAINEN AND EERO SAKSMAN
}

(Communicated by J. Marshall Ash)

\begin{abstract}
We prove that a complete metric space $X$ carries a doubling measure if and only if $X$ is doubling and that more precisely the infima of the homogeneity exponents of the doubling measures on $X$ and of the homogeneity exponents of $X$ are equal. We also show that a closed subset $X$ of $\mathbf{R}^{n}$ carries a measure of homogeneity exponent $n$. These results are based on the case of compact $X$ due to $\mathrm{Vol}^{\prime}$ berg and Konyagin.
\end{abstract}

\section{INTRODUCTION}

Let $X$ be a metric space. Denote by $B(x, r)$ the closed ball in $X$ with center $x$ and radius $r$. A Borel measure $\mu$ on $X$ is doubling if there is a constant $D \geq 1$ such that

$$
\mu(B(x, 2 r)) \leq D \mu(B(x, r)) \quad \text { for all } x \in X \text { and } r>0
$$

and if $\mu$ is nondegenerate in the sense that $\mu\left(B_{1}\right)>0$ and $\mu\left(B_{2}\right)<\infty$ for some balls $B_{1}$ and $B_{2}$ (if $X$ is nonempty). In this case $0<\mu(B)<\infty$ for all balls $B$, and

$$
\mu(B(x, \lambda r)) \leq c \lambda^{s} \mu(B(x, r)) \quad \text { for all } x \in X, r>0 \text {, and } \lambda \geq 1
$$

with $c=D$ and $s=\log _{2} D$. A nondegenerate Borel measure $\mu$ on $X$ satisfying (2) for some $c \geq 1$ and $s \geq 0$ is $(c, s)$-homogeneous, and it satisfies (1) with $D=2^{s} c$.

Coifman and Weiss $[\mathrm{CW}]$ observed that if there is a doubling measure on $X$ with constant $D$, then the metric space $X$ is doubling in the sense that there is a constant $N \in \mathbf{N}=\{1,2, \ldots\}$ depending only on $D$ such that for each $r>0$, each closed ball in $X$ of radius $2 r$ can be covered by a family of at most $N$ closed balls of radius $r$. In this case there are constants $C \geq 1$ and $s \geq 0$ depending only on $N$ such that if $x \in X, r>0$, and $\lambda \geq 1$, then the cardinality of every set in $B(x, \lambda r)$ whose points are at least $r$ apart is at most $C \lambda^{s}$. A metric space $X$ having this property for some $C \geq 1$ and $s \geq 0$ is $(C, s)$-homogeneous, and it is doubling with constant $N=\left\lfloor 2^{s} C\right\rfloor$. As easily shown by Vol'berg and Konyagin [VK2], a metric space carrying a $(c, s)$-homogeneous measure is $(C, s)$-homogeneous with $C$ depending only on $(c, s)$.

Two useful invariants of the metric space $X$ emerge. The Assouad dimension $\operatorname{dim}_{\mathrm{A}} X \in[0, \infty]$ of $X$ is the infimum of the numbers $s$ for which $X$ is

Received by the editors August 20, 1996.

1991 Mathematics Subject Classification. Primary 28A12; Secondary 54 F45.

Key words and phrases. Doubling metric space, homogeneous metric space, Assouad dimension, doubling measure, homogeneous measure.

(C) 1998 American Mathematical Society 
$(C, s)$-homogeneous for some $C$. In particular, Euclidean $n$-space $\mathbf{R}^{n}$ is $\left(C_{n}, n\right)$ homogeneous for some $C_{n}$ and has Assouad dimension $n$. The Vol'berg-Konyagin dimension $\operatorname{dim}_{\mathrm{VK}} X \in[0, \infty]$ of $X$ is the infimum of the numbers $s$ for which $X$ carries a $(c, s)$-homogeneous measure for some $c$. Thus, $\operatorname{dim}_{\mathrm{A}} X<\infty$ if and only if $X$ is doubling, $\operatorname{dim}_{\mathrm{VK}} X<\infty$ if and only if $X$ carries a doubling measure, and always

$$
\operatorname{dim}_{\mathrm{A}} X \leq \operatorname{dim}_{\mathrm{VK}} X .
$$

In [A2] Assouad asked whether conversely every doubling metric space carries a doubling measure, but he observed that not always: As $\mu(\{x\})=0$ whenever $\mu$ is a doubling measure on $X$ and $x$ is a nonisolated point in $X$, there are no doubling measures on the set of rational numbers. He then conjectured that complete doubling metric spaces carry a doubling measure. Working slightly later than Assouad but being unaware of him, Dyn'kin [D1], [D2] constructed a doubling measure on every compact set $X$ in $\mathbf{R}^{1}$ satisfying a porosity condition equivalent by [Lu] to $\operatorname{dim}_{\mathrm{A}} X<1$ and formulated Assouad's conjecture for closed sets in $\mathbf{R}^{n}$. Vol'berg and Konyagin [VK2] (results announced in [VK1]), motivated by Dyn'kin, established the conjecture for compact doubling metric spaces $X$, in the strong form that $\operatorname{dim}_{\mathrm{A}} X=\operatorname{dim}_{\mathrm{VK}} X$, and obtained for compact sets in $\mathbf{R}^{n}$ a sharp uniform result.

In this note we establish the conjecture for complete metric spaces in full, as an equality in (3), and also generalize the above-mentioned sharp uniform result for closed sets in $\mathbf{R}^{n}$. The argument is based on the compact case. Although it is simple, no proofs have appeared in literature as far as we know. Especially, in [VK1] the authors state their results for complete spaces and closed sets, but in [VK2] they prove only the compact case.

The invariant $\operatorname{dim}_{\mathrm{A}} X$ of $X$ was introduced by Assouad [A1], who called it the metric dimension $\operatorname{Dim} X$ of $X$, and by $\mathrm{Vol}^{\prime}$ berg and Konyagin [VK1], who called it the uniform metric dimension $\alpha(X)$ of $X$. Since the term metric dimension has often been used also for certain other dimension concepts (the lower and upper Minkowski dimensions), we prefer to call the present dimension concept just by the term Assouad dimension, named so after the first author who introduced this concept. The number $\operatorname{dim}_{\mathrm{VK}} X$ was introduced in [VK1] as the invariant $\beta(X)$.

Proofs of the elementary properties of Assouad dimension, more results about it, and a survey of the earlier ones can be found in [Lu]. Assouad dimension has proved in many ways to be a powerful invariant of metric spaces.

\section{Results}

Theorem 1. Let $C \geq 1, s \geq 0$, and $t>s$. Then there is $c \geq 1$ such that every $(C, s)$-homogeneous complete metric space carries a $(c, t)$-homogeneous measure.

Corollary. We have $\operatorname{dim}_{\mathrm{A}} X=\operatorname{dim}_{\mathrm{VK}} X$ for every complete metric space $X$.

Theorem 2. Let $n \in \mathbf{N}$. Then there is $c_{n} \geq 1$ such that every closed subset of $\mathbf{R}^{n}$ carries a $\left(c_{n}, n\right)$-homogeneous measure.

In the compact case these results are due to Vol'berg and Konyagin [VK2, Theorems 1 and 2]. For us it is crucial that a close examination of their proof yields the existence of the multiplicative constants $c$ and $c_{n}$, independent of the space. The same constants will persist in the general case. In [ $\mathrm{Lu}, 6.13]$ a proof is given for the compact case of Theorem 2 in order to correct a slight inaccuracy in [VK2]. 
Proof of Theorem 1 . Let $c$ be the constant given by Theorem 1 in the compact case. Let $X$ be a noncompact $(C, s)$-homogeneous complete metric space. We construct a $(c, t)$-homogeneous measure on $X$. Choose a point $x_{0} \in X$. If $k \in \mathbf{N}$, let $X_{k}=$ $B\left(x_{0}, k\right)$; then $X_{k}$ is $(C, s)$-homogeneous and bounded, thus totally bounded, and complete, hence compact. It follows that there is a $(c, t)$-homogeneous measure $\mu_{k}$ on $X_{k}$ normalized so that $\mu_{k}\left(X_{1}\right)=1$. Then $\mu_{k}\left(X_{p}\right) \leq c p^{t} \mu_{k}\left(X_{1}\right)=c p^{t}$ for all $p \in \mathbf{N}$ with $p \leq k$.

Consider $p \in \mathbf{N}$ and a subsequence $\left(\mu_{j}^{\prime}\right)_{j \in \mathbf{N}}$ of the sequence $\left(\mu_{k}\right)_{k \in \mathbf{N}}$. Let $C\left(X_{p}\right)$ be the supremum-normed Banach space of all continuous functions $f: X_{p} \rightarrow \mathbf{R}$. For $j \geq p$ define a linear functional $I_{j}: C\left(X_{p}\right) \rightarrow \mathbf{R}$ by setting $I_{j}(f)=\int_{X_{p}} f d \mu_{j}^{\prime}$ for $f \in C\left(X_{p}\right)$; then $\left\|I_{j}\right\|=\mu_{j}^{\prime}\left(X_{p}\right) \leq c p^{t}$. Since the closed unit ball of the conjugate space $C\left(X_{p}\right)^{*}$ of $C\left(X_{p}\right)$ endowed with the weak-* topology is compact [HR, B.25] and, as $C\left(X_{p}\right)$ is separable, metrizable, by considering the sequence $\left(I_{j} / c p^{t}\right)_{j \geq p}$ we obtain $J_{p} \in C\left(X_{p}\right)^{*}$ and a subsequence $\left(I_{j_{i}}\right)_{i \in \mathbf{N}}$ of the sequence $\left(I_{j}\right)_{j \geq p}$ such that $\lim _{i \rightarrow \infty} I_{j_{i}}(f)=J_{p}(f)$ for every $f \in C\left(X_{p}\right)$.

Thus, an induction on $p$ and Cantor's diagonal process yield a subsequence $\left(\mu_{j}^{*}\right)_{j \in \mathbf{N}}$ of $\left(\mu_{k}\right)_{k \in \mathbf{N}}$ such that $\left(\int_{X_{p}} f d \mu_{j}^{*}\right)_{j \geq p}$ is a convergent sequence for all $p \in \mathbf{N}$ and $f \in C\left(X_{p}\right)$. Let $C_{00}(X)$ be the linear space of all continuous functions $f: X \rightarrow \mathbf{R}$ whose support sp $f=\operatorname{cl}\{x \in X \mid f(x) \neq 0\}$ is compact. Now we can define a function $I: C_{00}(X) \rightarrow \mathbf{R}$ by setting

$$
I(f)=\lim _{p \leq j \rightarrow \infty} \int_{X_{p}} f d \mu_{j}^{*} \quad \text { if } f \in C_{00}(X), p \in \mathbf{N} \text {, and } \operatorname{sp} f \subset X_{p} .
$$

Then $I$ is linear and nonnegative: $I(f) \geq 0$ if $f \geq 0$. Hence, by F. Riesz's representation theorem [HR, 11.37] there is a Borel measure $\mu$ on $X$ such that $I(f)=\int_{X} f d \mu$ for every $f \in C_{00}(X)$ and such that $\mu(F)<\infty$ if $F \subset X$ is compact. We show that $\mu$ is the desired $(c, t)$-homogeneous measure. First, we have $\mu\left(X_{1}\right)<\infty$.

To establish (2) with $t$ replacing $s$, let $x \in X, r>0$, and $\lambda \geq 1$, and consider $\varepsilon \in(0,1)$. Choose $f, g \in C_{00}(X)$ such that $0 \leq f \leq 1, f \mid B(x, \lambda r)=1, \operatorname{sp} f \subset$ $B(x,(1+\varepsilon) \lambda r), 0 \leq g \leq 1, g \mid B(x,(1-\varepsilon) r)=1$, and sp $g \subset B(x, r)$. Choose $p \in \mathbf{N}$ with $B(x, 2 \lambda r) \subset X_{p}$. Pick $j \geq p$ so large that $\left|\int_{X_{p}} h d \mu-\int_{X_{p}} h d \mu_{j}^{*}\right|<\varepsilon$ if $h \in\{f, g\}$. Set $a_{\varepsilon}=c((1+\varepsilon) \lambda /(1-\varepsilon))^{t}$. Then we have

$$
\begin{aligned}
\mu(B(x, \lambda r)) & \leq \int_{X_{p}} f d \mu \leq \int_{X_{p}} f d \mu_{j}^{*}+\varepsilon \\
& \leq \mu_{j}^{*}(B(x,(1+\varepsilon) \lambda r))+\varepsilon \leq a_{\varepsilon} \mu_{j}^{*}(B(x,(1-\varepsilon) r))+\varepsilon \\
& \leq a_{\varepsilon} \int_{X_{p}} g d \mu_{j}^{*}+\varepsilon \leq a_{\varepsilon} \int_{X_{p}} g d \mu+a_{\varepsilon} \varepsilon+\varepsilon \leq a_{\varepsilon} \mu(B(x, r))+a_{\varepsilon} \varepsilon+\varepsilon .
\end{aligned}
$$

Now letting $\varepsilon \rightarrow 0$ this yields $\mu(B(x, \lambda r)) \leq c \lambda^{t} \mu(B(x, r))$.

Finally, to find a ball $B$ with $\mu(B)>0$, choose $f \in C_{00}(X)$ such that $0 \leq f \leq 1$, $f \mid X_{1}=1$, and $\operatorname{sp} f \subset X_{2}$. Then $\int_{X_{2}} f d \mu_{j}^{*} \geq \mu_{j}^{*}\left(X_{1}\right)=1$ for every $j \geq 2$. Hence, $\mu\left(X_{2}\right) \geq \int_{X_{2}} f d \mu \geq 1$.

Theorem 2 is proved in a similar manner.

Remarks. 1. We have $\operatorname{dim}_{\mathrm{A}} \tilde{X}=\operatorname{dim}_{\mathrm{A}} X$ and $\operatorname{dim}_{\mathrm{VK}} \tilde{X} \leq \operatorname{dim}_{\mathrm{VK}} X$ easily and then $\operatorname{dim}_{\mathrm{A}} X=\operatorname{dim}_{\mathrm{VK}} \widetilde{X}$ by the corollary for a metric space $X$ and its completion $\widetilde{X}$.

2. Theorem 2 does not hold for open subsets of $\mathbf{R}^{n}$. In fact, let $A \subset \mathbf{R}^{n}$ be a Cantor set of positive Lebesgue measure, and suppose that $X=\mathbf{R}^{n} \backslash A$ carries a 
$(c, n)$-homogeneous measure $\mu$ for some $c$; then $\mu$ extends to a $(c, n)$-homogeneous measure $\widetilde{\mu}$ on $\mathbf{R}^{n}$ with $\widetilde{\mu}(A)=0$, and thus the Lebesgue measure is not absolutely continuous with respect to $\widetilde{\mu}$, which is a contradiction by [J2, p. 2443].

3. Let $X$ be a noncomplete locally compact doubling metric space. We do not know whether $X$ always carries a doubling measure. (Added in proof: Solved in the negative by the second author.) However, if $X$ is $(C, s)$-homogeneous, so is $\tilde{X}$, and thus, if $t>s$, Theorem 1 provides a $(c, t)$-homogeneous measure $\mu$ on $\widetilde{X}$. Now, as $X$ is an open subspace of $\tilde{X}$, the restriction $\mu_{X}$ of $\mu$ to $X$ is a nondegenerate Borel measure on $X$, and for all $x \in X$ and $\lambda \geq 1$ it is easy to see that $\mu_{X}(B(x, \lambda r)) \leq c \lambda^{t} \mu_{X}(B(x, r))$ if $0<r \leq \operatorname{dist}(x, \widetilde{X} \backslash X)$.

4. In [Lu, 6.18] the compact case of Theorem 1 is generalized to a strictly different direction, namely to an analogue of Theorem 1 where the limitation $r \leq 1$ is imposed on the definitions of both the $(C, s)$-homogeneity of the space and the $(c, t)$ homogeneity of the measure; the measure can then be chosen to satisfy a uniform two-sided normalization condition for all balls of radius 1. A similar analogue of Theorem 2 is given in [J1] and [Lu, 6.19].

\section{REFERENCES}

[A1] P. Assouad, Étude d'une dimension métrique liée à la possibilité de plongements dans $\mathbf{R}^{n}$, C. R. Acad. Sci. Paris Sér. A 288 (1979), 731-734. MR 80f:54030

[A2] - Pseudodistances, facteurs et dimension métrique, Séminaire d'Analyse Harmonique 1979-1980, Publ. Math. Orsay 80, 7, Univ. Paris XI, Orsay, 1980, pp. 1-33. MR 84h:54027

[CW] R. R. Coifman and G. Weiss, Analyse harmonique non-commutative sur certains espaces homogènes, Lecture Notes in Math., vol. 242, Springer-Verlag, Berlin, 1971. MR 58:17690

[D1] E. M. Dyn'kin, Free interpolation by functions with derivatives in $H^{1}$, J. Soviet Math. 27 (1984), 2475-2481. (Russian) MR 84h:30052

[D2] Homogeneous measures on subsets of $\mathbf{R}^{n}$, Linear and complex analysis problem book (V. P. Havin, S. V. Hruščëv, and N. K. Nikol'skii, eds.), Lecture Notes in Math., vol. 1043, Springer-Verlag, Berlin, 1984, pp. 698-699.

[HR] E. Hewitt and K. A. Ross, Abstract harmonic analysis. I, Springer-Verlag, Berlin, 1963. MR 28:158

[J1] A. Jonsson, Besov spaces on closed subsets of $\mathbf{R}^{n}$, Trans. Amer. Math. Soc. 341 (1994), 355-370. MR 94c:46065

[J2] - Measures satisfying a refined doubling condition and absolute continuity, Proc. Amer. Math. Soc. 123 (1995), 2441-2446. MR 95j:28004

[Lu] J. Luukkainen, Assouad dimension: Antifractal metrization, porous sets, and homogeneous measures, manuscript, 1996.

[VK1] A. L. Vol'berg and S. V. Konyagin, There is a homogeneous measure on any compact subset in $\mathbf{R}^{n}$, Soviet Math. Dokl. 30 (1984), 453-456. (Russian) MR 86d:28018

[VK2] _ On measures with the doubling condition, Math. USSR-Izv. 30 (1988), 629-638. (Russian) MR 88i:28006

Department of Mathematics, P.O. Box 4 (Yliopistonkatu 5), Fin-00014 University of Helsinki, Finland

E-mail address: luukkain@cc.helsinki.fi

E-mail address: saksman@cc.helsinki.fi 